\title{
Robustness of Superconductivity to External Pressure in High-Entropy-Alloy-Type Metal Telluride AgInSnPbBiTe $_{5}$
}

Yoshikazu Mizuguchi ( $\nabla$ mizugu@tmu.ac.jp )

Tokyo Metropolitan University

Riad Kasem

Tokyo Metropolitan University

Yuki Nakahira

Tokyo Metropolitan University

Hitoshi Yamaoka

RIKEN

Ryo Matsumoto

National Institute for Materials Science

Aichi Yamashita

Tokyo Metropolitan University

Hirofumi Ishii

National Synchrotron Radiation Research Center

Nozomu Hiraoka

National Synchrotron Radiation Research Center

Yoshihiko Takano

National Institute for Materials Science

Yosuke Goto

Tokyo Metropolitan University

\section{Research Article}

Keywords:

Posted Date: February 23rd, 2022

DOI: https://doi.org/10.21203/rs.3.rs-1319304/v1

License: (c) (i) This work is licensed under a Creative Commons Attribution 4.0 International License.

Read Full License 


\section{Abstract}

High-entropy-alloy (HEA) superconductors are a new class of disordered superconductors. However, commonality of superconducting characteristics of HEA materials is unclear. Here, we have investigated the crystal and electronic structure, and the robustness of superconducting states in a HEA-type metal telluride ( $M T e ; M=\mathrm{Ag}, \mathrm{In}, \mathrm{Sn}, \mathrm{Pb}, \mathrm{Bi}$ ) under high pressure, and the results were compared with the pressure effects for a middle-entropy system $\left(\mathrm{AgPbBiTe}_{3}\right)$ and a reference system of PbTe. When the crystal structure is CsCl-type, all phases show superconductivity under high pressure but exhibit different pressure dependences of the transition temperature $\left(T_{\mathrm{C}}\right)$. For PbTe, its $T_{\mathrm{C}}$ decreases with pressure. In contrast, the $T_{\mathrm{c}}$ of HEA-type AgInSnPbBiTe$e_{5}$ is almost independent of pressure, for pressures ranging from 13.0 to $35.1 \mathrm{GPa}$. Those results suggest that the robustness of superconductivity to external pressure is linked to the configurational entropy of mixing at the $M$ site in $M T e$. Since the trend is quite similar to previous work on a HEA (Ti-Zr-Hf-Nb-Ta), where the robustness of superconductivity was observed up to $\sim 200 \mathrm{GPa}$, we propose that the robustness of superconductivity under high pressure would be a universal feature in HEA-type superconductors.

\section{Introduction}

The superconductivity of disordered materials has been extensively studied because of an observation made on the insulator-superconductor transition or disorder-induced superconductivity [1-3]. In addition, recent studies on $\mathrm{BiS}_{2}$-based layered superconductors have shown the importance of controlling the local structural disorder to improve superconducting properties [4-7]. As a new category of disordered superconductors, high-entropy alloys (HEAs), which are alloys containing five or more elements with an atomic concentration between $5 \%$ and $35 \%[8,9]$, have been extensively studied, leading to the discovery of a wide range of HEA superconductors [10-12]. One of the notable features is the difference in characteristics of superconductivity among conventional metal or alloy superconductors, superconducting thin films, and HEA superconductors $[11,13]$. Furthermore, the robustness of superconductivity to extremely high pressure (HP), for pressures up to $190 \mathrm{GPa}$, in an HEA, $(\mathrm{TaNb})_{0.67}(\mathrm{HfZrTi})_{0.33}$, was observed [14]. The high configurational entropy of mixing $\left(\Delta S_{\text {mix }}\right)$ reduces the Gibbs's free energy, and therefore stabilization of the phase is expected. The equation for calculating the Gibb's free energy is $\Delta G=\Delta H-T \Delta S_{\text {mix }}$, where $\Delta H$ is the enthalpy and $T$ is the absolute temperature. $\Delta S_{\text {mix }}$ can be calculated using the equation $\Delta S_{\text {mix }}=-R \sum_{i} c_{i} \mid \mathrm{n} c_{i}$, where, $R$ is the gas constant and $c_{i}$ is the atomic ratio of the element ( $(I)$ [8]. The discovery of the robustness of superconductivity under an extremely high pressure was considered to be related to the high $\Delta S_{\text {mix }}$, but similar robustness of superconductivity under an extremely high pressure and a high transition temperature $\left(T_{\mathrm{c}}\right)$ of $19 \mathrm{~K}$ was observed in a lowentropy $\mathrm{Nb}$-Ti alloy [15]. Therefore, the effects of $\Delta S_{\text {mix }}$ on the crystal structure, electronic structure, and superconducting properties of HEAs are still unclear. To address this issue, further investigation of HP effects on HEA-type materials is needed. 
We recently developed superconducting HEA-type compounds, in which one of the crystallographic sites is alloyed with the criterion similar to HEAs [16]. Since compounds with two or more crystallographic sites have unique chemical bonds, various (novel) effects of the introduction of HEA site to electronic and structural properties would be expected, which is so-called cocktail effect in the field of HEAs. In the layered $\mathrm{BiS}_{2}$-based superconductor $R E(\mathrm{O}, \mathrm{F}) \mathrm{BiS}_{2}$, the rare-earth site $(R E)$ was alloyed with five different $R E$ elements [17], and the improvement of superconducting properties (bulk nature of superconductivity) with increasing $\Delta S_{\text {mix }}$ was observed [18]. $T_{\mathrm{c}}$ was not affected by $\Delta S_{\text {mix }}$ and therefore its insensitivity to $\Delta S_{\text {mix }}$ in the $\mathrm{BiS}_{2}$-based superconductor is explained using a two-dimensional structure, since similar insensitivity was also observed in an RE123 cuprate with an HEA-type $R E$ site [19]. In contrast, $T_{\mathrm{c}}$ of $\mathrm{NaCl}$ type metal telluride, which is the target phase of this study, shows that its sensitivity to $\Delta S_{\text {mix }}$ of HEA-type systems is larger than that of metal tellurides with one or two constituent elements at the metal site [20, 21].

On the basis of the facts described above, we considered that the metal telluride system is suitable for the discussion of the effects of high $\Delta S_{\text {mix }}$ on the crystal structure (phase stability) and superconducting properties under high pressure. Here, we studied the crystal structure, electronic structure, and superconducting properties of three tellurides $(M T e ; M=\mathrm{Ag}, \mathrm{In}, \mathrm{Sn}, \mathrm{Pb}, \mathrm{Bi})$ with different $\Delta S_{\text {mix }}$ values at the $M$ site: $\operatorname{PbTe}\left(\Delta S_{\text {mix }}=0\right), \operatorname{AgPbBiTe}_{3}\left(\Delta S_{\text {mix }}=1.1 R\right)$, and $\operatorname{AgInSnPbBiTe}{ }_{5}\left(\Delta S_{\text {mix }}=1.6 R\right)$. The pressure evolutions of the crystal structure and electronic transport properties were reported in previous studies [22-26]. Although PbTe is a semiconductor under ambient and low pressures, it undergoes metallization under high pressures, and a superconducting transition is observed for pressures above $15 \mathrm{GPa}[22,23]$. $\mathrm{PbTe}$ undergoes structural transitions as follows: cubic NaCl-type $(F m-3 m)$ structure under low pressures, orthorhombic (Pnma) structure under moderate pressures, and cubic CsCl-type ( $P m-3 m)$ structure under $\mathrm{HP}$ [24-26]. There is a report on synthesis and thermoelectric properties of $\mathrm{AgPbBiTe}_{3}$, but the structural and physical properties of $\mathrm{AgPbBiTe}_{3}$ have not been reported [27]. In this study, we established a phase diagram of the crystal structures, as well as one for superconductivity versus pressure for both $\mathrm{AgPbBiTe}_{3}$ and $\mathrm{AgSnInPbBiTe}{ }_{5}$. We also studied PbTe to examine the effects of configurational entropy of mixing. The pressure phase diagrams for the three tellurides are used to compare the crystal and electronic structures in detail. The results show that the pressure phase diagram of the crystal structure is largely modified by the effect of $\Delta S_{\text {mix }}$. Furthermore, we found that the pressure dependence of $T_{\mathrm{c}}$ in the HP phase (CsCl-type phase) exhibits a clear difference among the three compounds. In the HP phase, the $T_{\mathrm{c}}$ for $\mathrm{PbTe}$ decreases with pressure, but that for HEA-type AgInSnPbBiTe ${ }_{5}$ exhibits a flat dependence, which suggests that the superconducting state of $\mathrm{AgInSnPbBiTe}$ is robust to pressure. Our present findings show an analogical conclusion in the case of $(\mathrm{TaNb})_{0.67}(\mathrm{HfZrTi})_{0.33}[14]$ and suggest that the HEA effects universally enhance the robustness of superconductivity under high pressure.

\section{Results}

\subsection{Electrical resistance}


Figure 1a shows the temperature dependence of the electrical resistance of $\mathrm{AgInSnPbBiTe}{ }_{5}$ measured using the conventional four-probe method at ambient pressure. $T_{\mathrm{c}}^{\text {zero }}$ was $1.8 \mathrm{~K}$, which is slightly lower than that reported in previous research [20]. We measured the electrical resistance (at National Institute for Materials Science) after several days since the sample was synthesized by HP annealing (at Tokyo Metropolitan University), and a slight aging effect of $T_{\mathrm{C}}$ was noticed in $M T e$ superconductors synthesized under high pressure $[28,29]$. We consider that the slight decrease in $T_{\mathrm{C}}$ can be understood by the change in the internal strains because the XRD patterns do not show a remarkable change after aging in the HPsynthesized MTe samples. Therefore, we continued to perform resistance measurements under high pressure using a diamond-anvil cell (DAC).

Figure $1 \mathrm{~b}$ shows the temperature dependences of the electrical resistance of AglnSnPbBiTe ${ }_{5}$ measured under various pressures with a DAC. Normal-state resistance decreases with pressure, for pressures up to 9.3 $\mathrm{GPa}$ and increases with pressure for pressures above 15.5 GPa. An onset of the superconducting transition was observed at $2 \mathrm{~K}$ under $6.5 \mathrm{GPa}$, and the zero-resistance state was observed at $T_{\mathrm{c}}^{\text {zero }}=2 \mathrm{~K}$ under $10.2 \mathrm{GPa}$. As shown in Fig. $2 \mathrm{a}$, the $T_{\mathrm{c}}{ }^{\text {onset }}$ monotonously increased with pressure until it reached 13.0 $\mathrm{GPa}$, and then it became insensitive to pressure for pressures ranging from $13.0 \mathrm{GPa}$ to $35.1 \mathrm{GPa}(\mathrm{P}$ $=35.1 \mathrm{GPa}$ is the chosen maximum pressure for the experiment). The results indicate that the $T_{\mathrm{c}}$ in the $\mathrm{CsCl}$-type structure of $\mathrm{AgInSnPbBiTe}_{5}$ is independent of applied pressure, while lattice constant decreases with pressure. The highest $T_{\mathrm{c}}$ zero and $T_{\mathrm{c}}$ onset observed in the experiment on AgInSnPbBiTe 5 are $4.5 \mathrm{~K}$ and $5.3 \mathrm{~K}$, respectively. See Figure $\mathrm{S} 1$ for the determination criterion for $T_{\mathrm{c}}$ onset.

To compare the pressure evolution of $T_{\mathrm{c}}$ in $\mathrm{AgInSnPbBiTe}{ }_{5}$ with that of $\mathrm{PbTe}$ and $\mathrm{AgPbBiTe}$, the pressure dependences of $T_{\mathrm{c}}$ onset for $\mathrm{PbTe}$ and $\mathrm{AgPbBiTe}_{3}$ were examined (see Fig. 2c for structural difference and Figs. S2 and S3 for the resistance measurements), and the resulting $T_{\mathrm{c}}-P$ plots are shown in Fig. $2 \mathrm{~b}$. PbTe is a semiconductor at ambient and low pressures, but exhibits a pressure-induced superconducting transition above $17 \mathrm{GPa}$ in the $\mathrm{CsCl}$-type structure [22]. At higher pressures, $T_{\mathrm{c}}$ of $\mathrm{PbTe}$ monotonously decreases with pressure. In the case of $\mathrm{AgPbBiTe}_{3}$, superconductivity was observed at $P>2.6 \mathrm{GPa}$, and at this instance $T_{\mathrm{c}}$ onset reached $6.5 \mathrm{~K}$. The $T_{\mathrm{c}}$ for $\mathrm{AgPbBiTe}_{3}$ slightly decreases at high pressures. In AgInSnPbBiTe ${ }_{5}$, superconductivity is observed at low pressures as well because the low-pressure phase, having a $\mathrm{NaCl}$-type structure, itself is a metal and shows superconductivity under ambient pressure (Figs. 1a and 2a). As demonstrated in the next section, the crystal-structure type under high pressure is $\mathrm{CsCl}$-type for all the compounds. However, the trend of the pressure dependences of $T_{\mathrm{c}}$ in the CsCl-type structure exhibit a clear difference among $\mathrm{PbTe}, \mathrm{AgPbBiTe}_{3}$, and $\mathrm{AgInSnPbBiTe} e_{5}$. The main findings of this study are that the robustness of superconductivity to pressure in HEA-type AgInSnPbBiTe $e_{5}$ is similar to that observed in $(\mathrm{TaNb})_{0.67}(\mathrm{HfZrTi})_{0.33}$ [14]. To validate the conclusion, we investigated the pressure evolutions of the crystal structure and the electronic structure for those MTe samples under high pressure.

\subsection{Crystal structure}


Figures $3 a-3 c$ show the pressure-dependent synchrotron X-ray diffraction (SXRD) patterns for the PbTe, $\mathrm{AgPbBiTe}_{3}$, and AgInSnPbBiTe ${ }_{5}$ samples, respectively. For all the SXRD patterns, we performed the Rietveld refinement to confirm the structural type and to evaluate the lattice constant. See Tables S1-S3 and Figs. S4-S6 for details on refinements. On the basis of the refinement results, we established structural phase diagrams under high pressure (Figs. 3d-3f) by plotting the pressure dependence of volume per unit formula ( $Z$ ). For $\mathrm{PbTe}$, the structural transition from $\mathrm{NaCl}$-type to Pnma occurs at around $6.80 \mathrm{GPa}$, and the second transition to $\mathrm{CsCl}$-type takes place at $14.28 \mathrm{GPa}$. The transition gradually occurred, hence the phase diagram contains mixed phases. The results on $\mathrm{PbTe}$ are consistent with the previous work by Li et al. [24]. For $\mathrm{AgPbBiTe}_{3}$ and $\mathrm{AgInSnPbBiTe}_{5}$, similar phase diagrams were obtained, where the NaCl-type structure is stabilized up to $~ 10 \mathrm{GPa}$, and the Pnma phase is suppressed. The pressure where the $\mathrm{CsCl}$-type phase is induced is common to the case of $\mathrm{PbTe}$. In all the structural types including the $\mathrm{CsCl}$-type phase, the lattice volume continuously decreases with pressure. Although the difference in the stability of the $\mathrm{NaCl}$-type and Pnma structures may be related to the difference in lattice volume at ambient pressure, we consider that the Pnma phase is suppressed, and the NaCl-type phase is stabilized by the effect of alloying at the $M$ site. We note that configurational entropy of mixing does not affect the structure of the CsCl-type phase, and lattice volume of the CsCl-type phase commonly decreases with pressure in three MTe sample.

\subsection{Electronic structure}

To examine the effects of pressure and HEA states on the electronic structure, we performed X-ray absorption spectroscopy with partial fluorescence mode (PFY-XAS) for PbTe and AgInSnPbBiTe ${ }_{5}$. See Fig. $S 7$ for pressure dependences of spectra, and analysis results on the $\mathrm{Pb}-L_{3}$ and $\mathrm{Bi}-L_{3}$ spectra. In general, the absorption spectra at the $\mathrm{Pb}-L_{3}$ absorption edge are similar to those at the $\mathrm{Bi}-L_{3}$ absorption edge. On the basis of analogically referring to other $\mathrm{Pb}$ - or Bi-containing compounds [30,31], we analyzed the spectra by assuming several peaks. An example of the fit for the PFY-XAS spectra at $27 \mathrm{GPa}$ is shown in Fig. 4a. The PFY-XAS spectra were fitted by assuming some Voigt functions with an arctan-like background [31]. In this study, we focus on the peaks of P1, P2, and P3, where peak P1 could be assigned as a dipole transition of the $2 p_{3 / 2}$ electron into the $6 s$ state, and the peaks shown as P2 and P3 correspond to $6 d$ states of $t_{2 g}$ and $e_{g}$, respectively [32,33]; we measure $2 p_{3 / 2} \rightarrow n d(n>6)$ transitions at the $\mathrm{Pb} L_{3}$ absorption edge. There is a $p$ density of states ( $p$ DOS) above the Fermi level, however, we mainly observe the dipole-allowed transitions of $\mathrm{Pb} 2 p-6 s$ and $\mathrm{Pb} 2 p-6 d$, and therefore, the observed spectra do not reflect the $\mathrm{Pb} 6 p$ DOS spectroscopically. The absorption spectra reflect the empty DOS above the Fermi level generally, with a core hole in the final state.

For $\mathrm{PbTe}$ with a $\mathrm{NaCl}$-type structure, there is a narrow band gap, and the $p$ orbitals of $\mathrm{Pb}$ and Te near the Fermi energy are hybridized. See Figure S8 for the calculated DOS for PbTe [34]. The valence band is mainly composed of Te $5 p$ orbitals, and also contains the contribution from $\mathrm{Pb} 6 p$ and $6 s$, while the conduction band is mainly composed of $\mathrm{Pb} 6 p$ orbitals, but also contains $\mathrm{Te} 5 p$ contributions. For $\mathrm{PbTe}$ with a CsCl-type structure, band gap is totally closed, and the DOS near the Fermi energy is explained by 
the contributions from $\mathrm{Pb} 6 p$ and $6 s$ orbitals, as well as the Te $5 p$ orbitals. Therefore, the pressure evolution of the $6 \mathrm{~s}$ states which could be resolved in our high-resolution spectroscopy, may play an important role on the closing of the band gap as well as the emergence of superconductivity.

The pressure dependences of the intensity and the energy of peak $\mathrm{P} 1$ in PbTe is shown in Fig. 4b. The intensity of peak P1 in Fig. 4b gradually increases with pressure, up to about $5 \mathrm{GPa}$. The increase in the intensity of $\mathrm{P} 1$ indicates an increase in the amount of holes in the $\mathrm{Pb} 6 s$ states, which indicates a modification of the band structure. In the middle-pressure phase, (between 5-15 GPa) the intensity of P1 does not show a significant change, while it increases remarkably in the HP phase ( $P>15 \mathrm{GPa})$. We note that $\mathrm{PbTe}$ with a $\mathrm{NaCl}$-type or Pnma structure is a semiconductor with a band gap at the low-pressure regime, and we also note that the metallic phase is induced in a CsCl-type structure for pressures above $15 \mathrm{GPa}[22,34,35]$. The increase of the intensity of P1 corresponds with the increase of the unoccupied $6 s$ states of the $\mathrm{Pb}$. This may correlate with the emergence of the superconductivity after the closing of the band gap at $P>15 \mathrm{GPa}$.

On the other hand, the energy of peak $\mathrm{P} 1$ shifts to a lower incident energy until it reaches a pressure level of $5 \mathrm{GPa}$. The incident energy and the intensity do not change in the middle-pressure range of $5-17 \mathrm{GPa}$, and they start decreasing again for pressures above $18 \mathrm{GPa}$ as shown in Fig. $4 \mathrm{~b}$. The shift of the energy of peak P1 to a lower incident energy is explained by the upward shift of the Fermi level or change in the DOS at the Fermi level. Theory suggests that the energy shift of peak P1 may be influenced by the reduction of the band gap [35] and the theoretical band gap is in the same order as the energy shift of P1 at $5 \mathrm{GPa}$.

The intensity of $\mathrm{P} 3\left(\mathrm{~Pb} 6 d \mathrm{DOS}, e_{\mathrm{g}}\right)$ shows a trend which is similar to the intensity of P1. The intensity of $\mathrm{P} 2\left(\mathrm{~Pb} 6 d \mathrm{DOS}, t_{2 \mathrm{~g}}\right.$ ) on the other hand decreases with increasing pressure at $P>17 \mathrm{GPa}$ (Fig. 4c). It is interesting that there is a large change in the electronic structure for pressures above $20 \mathrm{GPa}$, but the crystal structure still retains its $\mathrm{CsCl}$-type structure in this pressure range. In $\mathrm{PbTe}$, superconductivity suddenly appears above $18 \mathrm{GPa}$, and $T_{\mathrm{C}}$ decreases with pressure monotonically [22]. The present result possibly suggests that the change in the electronic structure is not favorable for the superconductivity of MTe, when it has transitioned to the CsCl-type structure.

We also measured the PFY-XAS spectra at the Pb- $L_{3}$ absorption edges for AglnSnPbBiTe $e_{5}$ as shown in Fig. S7. We observed similar trends in the pressure dependence of the electronic structures as those observed for PbTe. An example of the fit at $28.8 \mathrm{GPa}$ is shown in Fig. 4d. The analysis results on P1, P2, and $\mathrm{P} 3$ are plotted in Figs. $4 \mathrm{e}$ and $4 \mathrm{f}$. Figure $4 \mathrm{e}$ shows a gradual increase of the P1 intensity with pressure, which is similar to the case of $\mathrm{PbTe}$. The trend of $\mathrm{P} 2$ is also similar for the entire pressure range, and that of P3 is basically similar between PbTe and AgInSnPbBiTe ${ }_{5}$. The PFY-XAS spectra at the Bi- $\angle 3$ absorption edge were also taken, and the analysis results are summarized in Fig. S7. In AgInSnPbBiTe the pressure-induced change in the electronic structure seems to be common for $\mathrm{Bi}$ and $\mathrm{Pb}$ sites; the detailed results are shown under the Supporting Information section. In conclusion, the electronic structures of $\mathrm{PbTe}$ and $\mathrm{AgInSnPbBiTe} e_{5}$ show a similar pressure dependence, even though the structure of 
$\mathrm{PbTe}$ does not change much in the middle-pressure range (Pnma $+\mathrm{CsCl}$ phase), which disappears in $\mathrm{AgInSnPbBiTe}_{5}$. Therefore, the difference in the robustness of superconductivity to pressure in the $\mathrm{CsCl}-$ type phase between $\mathrm{PbTe}$ and $\mathrm{AgInSnPbBiTe}{ }_{5}$ cannot be explained by the pressure evolutions of crystal and electronic structures.

\section{Discussion}

From the structural viewpoint, the impact of the introduction of an HEA site is the suppression of the middle-pressure phase with a Pnma structure. In other words, the low-pressure phase with a NaCl-type structure is stabilized up to a higher pressure of $P>10 \mathrm{GPa}$ in AglnSnPbBiTe${ }_{5}$, whereas the $\mathrm{NaCl}$-type phase disappears at $\sim 5 \mathrm{GPa}$ for PbTe. Interestingly, the trend of lattice constant in the CsCl-type structure under $\mathrm{HP}$ is quite similar for $\mathrm{PbTe}$ and $\mathrm{AgInSnPbBiTe}{ }_{5}$. However, as revealed in Fig. 2b, the pressure dependences of $T_{\mathrm{c}}$ clearly differ since $T_{\mathrm{c}}$ decreases with pressure for PbTe but does not change largely in the $\mathrm{CsCl}$-type phase for $\mathrm{AgInSnPbBiTe}_{5}$. Furthermore, from the electronic-structure viewpoint, we cannot find a clear correlation between the robustness of superconductivity to pressure and the changes in electronic structure under high pressure. Although there is a possibility of the difference of the contribution of the $6 p$ states, which could not be measured in our spectra, to the conduction band to increase the number of the carriers in $\mathrm{AgInSnPbBiTe}_{5}$.

The results show that the pressure phase diagram of the crystal structure is largely modified by the effect of $\Delta S_{\text {mix }}$. In contrast, the electronic structures are not sensitive to the effect of $\Delta S_{\text {mix }}$. To understand the HEA effects on structural and electronic properties for $M T e$ under high pressure, further studies using various probes are needed. However, commonality on the robustness of superconductivity to external pressure in the superconducting $\mathrm{HEA}(\mathrm{TaNb})_{0.67}(\mathrm{HfZrTi})_{0.33}[14]$ and the HEA-type metal telluride AglnSnPbBiTe ${ }_{5}$ would be demonstrating the universal characteristics of superconductivity in HEA-type materials. Thus, the present results propose that the combination of HP and HEA effects will open a new pathway to the development of new disordered superconductors with exotic superconducting states.

\section{Conclusion}

We have studied the crystal and electronic structure and the robustness of superconducting states in a HEA-type metal telluride ( $M T e ; M=\mathrm{Ag}, \mathrm{In}, \mathrm{Sn}, \mathrm{Pb}, \mathrm{Bi}$ ) under high pressure using a polycrystalline sample, and the results were compared with the pressure effects for a middle-entropy system $\left(\mathrm{AgPbBiTe}_{3}\right)$ and a reference system of $\mathrm{PbTe}$. $\mathrm{PbTe}$ exhibits a structural transition from a $\mathrm{NaCl}$-type to an orthorhombic Pnma structure at low pressures, and further transitions to a CsCl-type structure at high pressures. When the superconductivity of the $\mathrm{CsCl}$-type $\mathrm{PbTe}$ is observed, it is found that its superconducting transition temperature $\left(T_{\mathrm{c}}\right)$ decreases with pressure. In contrast, in the HEA-type AgInSnPbBiTe ${ }_{5}, T_{\mathrm{c}}$ is almost independent of pressure, for pressures ranging from 13.0 to $35.1 \mathrm{GPa}$. In addition, the middle-entropy system, $\mathrm{AgPbBiTe}_{3}$, shows a slight decrease in $T_{\mathrm{c}}$ with pressure in the $\mathrm{CsCl}$-type structure, which is intermediate trend between $\mathrm{PbTe}$ and $\mathrm{AgInSnPbBiTe}{ }_{5}$. Those results suggest that the robustness of 
superconductivity to external pressure has been enhanced by the increase in configurational entropy of mixing at the metal $(M)$ site in $M T e$. To further clarify the effects of the modification of the configurational entropy of mixing on the superconducting states and the electronic structure of $M T e$, synchrotron X-ray absorption spectroscopy with partial fluorescence mode (PFY-XAS) for three MTe polycrystalline samples of $\mathrm{PbTe}$ and $\mathrm{AgInSnPbBiTe}_{5}$ were performed. Noticebly, the evolutions of electronic structure under high pressure do not largely differ between $\mathrm{PbTe}$ and $\mathrm{AgInSnPbBiTe}$; ; hence, the difference in the robustness of superconductivity in $\mathrm{PbTe}$ and $\mathrm{AgInSnPbBiTe}$ under high pressure is not explained by the difference in their electronic structure. According to the results of this work and previous work on a HEA (Ti-Zr-Hf-Nb-Ta), where the robustness of superconductivity was observed up to $\sim 200 \mathrm{GPa}$, we propose that the robustness of superconductivity under high pressure would be a universal feature in HEA-type superconductors.

\section{Methods}

The polycrystalline sample of $\mathrm{PbTe}$ was synthesized by the solid-state reaction of $\mathrm{Pb}(99.9 \%)$ and $\mathrm{Te}$ $(99.999 \%)$ at $900 \otimes C$. To obtain a pellet for resistance measurements, pelletizing and second annealing were performed. The polycrystalline samples of $\mathrm{AgPbBiTe}_{3}$ and $\mathrm{AgInSnPbBiTe}_{5}$ were synthesized using

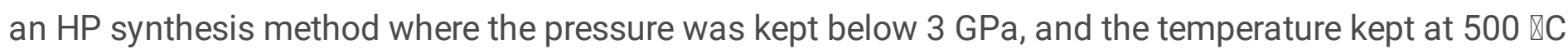
for 30 minutes as described in Ref. 20. The precursor powders of $\mathrm{AgPbBiTe}_{3}$ and $\mathrm{AgInSnPbBiTe}_{5}$ were synthesized by a solid-state reaction of Ag powders (99.9\%) and grains of In (99.99\%), Sn (99.999\%), $\mathrm{Pb}$ (99.9\%), Bi (99.999\%), and Te (99.999\%) at $800 \bowtie \mathrm{C}$, with the nominal compositions.

The electrical resistance measurements were performed at ambient pressure using the conventional fourprobe method on a GM refrigerator system. Resistance measurements under high pressure were performed on polycrystalline powder on a Physical Property Measurement System (Quantum Design) using an originally designed diamond anvil cell (DAC) with boron-doped diamond electrodes [36-38]. The sample was placed on the boron-doped diamond electrodes in the center of the bottom anvil. The surface of the bottom anvil, except for the sample space and electrical terminal, were covered with the undoped diamond insulating layer. The cubic boron nitride powders with ruby manometer were used as a pressuretransmitting medium. The applied pressure was estimated by the fluorescence from ruby powders [39] and the Raman spectrum from the culet of top diamond anvil [40] using an inVia Raman microscope (RENISHAW). The definition of $T_{\mathrm{c}}$ is described in Fig. S1.

Pressure dependences of the synchrotron X-ray powder diffraction (SXRD) patterns were measured at BL12B2, SPring-8, using a 3-pin plate diamond anvil cell (DAC, Almax easyLab Industries) with a CCD detection system at room temperature $(\sim 293 \mathrm{~K})$. Culet size of the diamond anvil was $0.4 \mathrm{~mm}$ with a stainless-steel gasket. We took an arrangement of both incoming and outgoing $\mathrm{x}$-ray beams passing through the diamonds with incident photon energy of $18 \mathrm{keV}$. A two-dimensional image of the CCD system was integrated using the FIT2D program [41]. Silicone oil was used as a pressure-transmitting 
medium, and pressure was monitored using the ruby fluorescence method. [42] The SXRD data was analyzed through Jana 2006 software [43] using the Rietveld method.

The pressure dependence of the high-resolution X-ray absorption spectra was measured at beamline BL12XU, SPring-8. Membrane-controlled DACs with a $0.3 \mathrm{~mm}$ culet and that with a $0.4 \mathrm{~mm}$ culet were used for $\mathrm{PbTe}$ and $\mathrm{AgInSnPbBiTe}{ }_{5}$, respectively, and silicone oil was used as a pressure-transmitting medium. Beryllium gaskets with a $3 \mathrm{~mm}$ diameter were pre-indented at the center. The thickness was approximately $67 \mu \mathrm{m}$ for $\mathrm{PbTe}$ and $31 \mu \mathrm{m}$ for $\mathrm{AgInSnPbBiTe}_{5}$, and the diameters of the sample chamber in the gaskets were approximately $110 \mu \mathrm{m}$ for PbTe and $140 \mu \mathrm{m}$ for AgInSnPbBiTe${ }_{5}$. We employed X-ray absorption spectroscopy (XAS) with a partial fluorescence mode (PFY-XAS), which has an advantage of a higher resolution as compared to that of normal XAS [44, 45]. We used the Be gasket in plane geometry where both incoming and outgoing $x$-ray beams passed through the Be gasket. A Johann-type spectrometer equipped with a spherically bent $\mathrm{Si}(555)$ analyzer crystal (radius of $\sim 1 \mathrm{~m}$ ), and a Si solid state detector were used to analyze the $\mathrm{Bi} L \mathrm{a}_{1}\left(10.839 \mathrm{keV}, 3 d_{5 / 2}-2 p_{3 / 2}\right)$ emission at the $\mathrm{Bi} L_{3}$ absorption edge, and $\mathrm{Pb} L a_{1}\left(10.551 \mathrm{eV}, 3 d_{5 / 2}-2 p_{3 / 2}\right)$ emission at the $\mathrm{Pb} L_{3}$ absorption edge [46]. The incident beam is focused at $17 \mu \mathrm{m} \times 40 \mu \mathrm{m}$ by the K-B mirror located at the sample position.

\section{Declarations}

\section{Acknowledgements}

The measurements of the XRD patterns and the PFY-XAS spectra under pressure were performed BL12XU, and BL12B2, SPring-8 under SPring-8 Proposal Nos. 2020A4269, 2021A4253, \& 2021B4254 (corresponding to Proposal Nos. 2019-2-261, 2021-1-009, 2021-1-404, \& 2021-1-415 of NSRRC).The authors thank O. Miura for his supports in experiments. This work was partially supported by Grant-in-Aid for Scientific Research (KAKENHI) (Nos. 18KK0076, 21K18834, 21 H00151) and Tokyo Metropolitan Government Advanced Research (H31-1). We thank Editage for English correction of the manuscript.

\section{Author contributions}

M.R.K., R.M., H.Y., Y.G. and Y.M. designed the research; M.R.K., A.Y., and Y.M. synthesized samples; R.M. and Y.T. performed resistance measurement; H.Y., H.I., and N.H. performed synchrotron experiments and analyzed the spectra of PFY-XAS; M.R.K., Y.N., A.Y., Y.G., and Y.M. analyzed the crystal structure; M.R.K., Y.N., H.Y., and Y.M. wrote the manuscript.

\section{Competing interests}

The Authors declare no Competing Financial or Non-Financial Interests

\section{Data availability}


The data that support the findings of this study are available from the corresponding author (Y.M.) upon reasonable request.

\section{References}

1. Dubi, Y., Meir, Y. \& Y. Avishai.Nature of the superconductor-insulator transition in disordered superconductors, Nature 449, 876 (2007).

2. Gastiasoro, M. N. \& Andersen, B. M. Enhancing superconductivity by disorder, Phys. Rev. B 98, 184510 (2018).

3. Zhao, K. et al. Disorder-induced multifractal superconductivity in monolayer niobium dichalcogenides, Nat. Phys. 15, 904 (2019).

4. Mizuguchi, Y. Material Development and Physical Properties of $\mathrm{BiS}_{2}$-Based Layered Compounds, Phys. Soc. Jpn. 88, 041001 (2019).

5. Mizuguchi, Y. et al.Evolution of Anisotropic Displacement Parameters and Superconductivity with

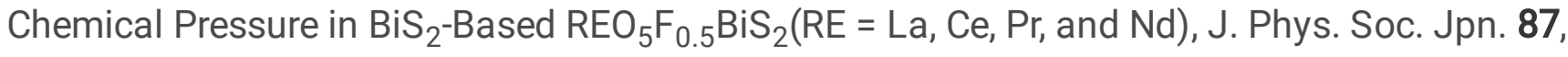
023704 (2018).

6. Paris, E. et al.Suppression of structural instability in $\mathrm{LaOBiS}_{2-} x$ Sexby Se substitution, J. Phys.: Condens. Matter 30, 455703 (2018).

7. Nagasaka, K. et al.Intrinsic Phase Diagram of Superconductivity in the $\mathrm{BiCh}_{2}$-Based System Without In-Plane Disorder, J. Phys. Soc. Jpn. 86, 074701 (2017).

8. Yeh, J. W. et al.Nanostructured High-Entropy Alloys with Multiple Principal Elements: Novel Alloy Design Concepts and Outcome, Adv. Energy Mater. 6, 299 (2004).

9. Ye, Y. F. et al.High-entropy alloy: challenges and prospects, today 19, 349 (2016).

10. Koželj, P. et al.Discovery of a Superconducting High-Entropy Alloy, Rev. Lett. 113, 107001 (2014).

11. Sun, L. \& Cava, R. J. Phys. High-entropy alloy superconductors: Status, opportunities, and challenges, Phys. Materials 3, 090301 (2019).

12. Kitagawa, J., Hamamoto, S. \&lshizu, N. Cutting Edge of High-Entropy Alloy Superconductors from the Perspective of Materials Research, Metals 10, 1078 (2020).

13. Stolze, K. et al.High-entropy alloy superconductors on an a-Mn lattice, Mater. Chem. C 6, 10441 (2018).

14. Guo, J. et al. Robust zero resistance in a superconducting high-entropy alloy at pressures up to 190 GPa, PNAS 114, 13144 (2017).

15. Guo, J. et al. Record-High Superconductivity in Niobium-Titanium Alloy, Adv. Mater. 33, 1807240 (2019).

16. Mizuguchi, Y. \& Yamashita, A. Superconductivity in HEA-Type Compounds, IntechOpen (2021).

17. Sogabe, R., Goto, Y. \&Mizuguchi, Y. Superconductivity in $\mathrm{REO}_{5} \mathrm{~F}_{0.5} \mathrm{BiS}_{2}$ with high-entropy-alloy-type blocking layers, Appl. Phys. Express 11, 053102 (2018). 
18. Sogabe, R. et al.Improvement of superconducting properties by high mixing entropy at blocking layers in $\mathrm{BiS}_{2}$-based superconductor $\mathrm{REO}_{5} \mathrm{~F}_{0.5} \mathrm{BiS}_{2}$, Solid State Commun. 295, 43 (2019).

19. Shukunami, Y. et al.Synthesis of RE123 high- $T_{C}$ superconductors with a high-entropy-alloy-type RE site, Physica C 572, 1353623 (2020).

20. Mizuguchi, Y. Superconductivity in High-Entropy-Alloy Telluride AgInSnPbBiTe ${ }_{5}$, J. Phys. Soc. Jpn. 88, 124708 (2019).

21. Kasem, Md. R. et al.Superconducting properties of high-entropy-alloy tellurides M-Te (M: Ag, In, Cd, $\mathrm{Sn}, \mathrm{Sb}, \mathrm{Pb}, \mathrm{Bi}$ ) with a NaCl-type structure, Phys. Express 13, 033001 (2020).

22. Brandt, N. B. et al.Superconductivity of the compounds $\mathrm{PbTe}$ and $\mathrm{PbSe}$ under high pressure, JETP Lett. 22, 104 (1975).

23. Xu, L., Zheng, Y. \& Zheng, J. C.Thermoelectric transport properties of PbTe under pressure, Rev. B 82, 195102 (2010).

24. Li, Y. et al.Phase transitions in PbTe under quasi-hydrostatic pressure up to $50 \mathrm{GPa}$, High Press. Res. 33, 713 (2013).

25. Fujii, Y. et al. A new high-pressure phase of PbTe above 16 GPa, Solid State Commun. 49, 135 (1984).

26. Bencherif, Y. et al. Chem. Phys. 126, 707 (2011).

27. Sportouch, S. et al. THERMOELECTRIC PROPERTIES OF THE CUBIC FAMILY OF COMPOUNDS $\mathrm{AgPbBiQ}_{3}(\mathrm{Q}=\mathrm{S}, \mathrm{Se}, \mathrm{Te})$. VERY LOW THERMAL CONDUCTIVITY MATERIALS, Mater. Res. Soc. Symp. Proc. 545, 123 (1999).

28. Katsuno, M. et al. High-Pressure Synthesis and Superconducting Properties of NaCl-Type $\ln _{1-x} P \mathrm{~Pb}_{x} \mathrm{Te}$ $(x=0-0.8)$, Condens. Matter 5, 14 (2020).

29. Mitobe, T. et al. Superconductivity in In-doped $\mathrm{AgSnBiTe}_{3}$ with possible band inversion, Rep. 11, 22885 (2021).

30. Swarbrick, J. C. et al.High Energy Resolution X-ray Absorption Spectroscopy of Environmentally Relevant Lead (II) Compounds, Chem. 48, 10748 (2009).

31. Yamaoka, $\mathrm{H}$. et al.Electronic structures of $\mathrm{Bi}_{2} \mathrm{Se}_{3}$ and $\mathrm{Ag}_{\mathrm{x}} \mathrm{Bi}_{2} \mathrm{Se}_{3}$ under pressure studied by highresolution x-ray absorption spectroscopy and density functional theory calculations, Rev. B 102, 155118 (2020).

32. Rao K. J. \& Wong, J. A XANES investigation of the bonding of divalent lead in solids, Chem. Phys. 81, 4832 (1984).

33. Retoux, R. et al. Valence state for bismuth in the superconducting bismuth cuprates, Rev. B 41, 193 (1990).

34. We obtained the electronic band structures of PbTe using CompES-X, NIMS database (https://compes-x.nims.go.jp/)

35. Xu, L., Zheng, Y. \& Zheng, J. C. Thermoelectric transport properties of PbTe under pressure, Rev. B 82, $195102(2010)$. 
36. Matsumoto, R. et al. Pressure-Induced Superconductivity in Sulfur-Doped SnSe Single Crystal Using Boron-Doped Diamond Electrode-Prefabricated Diamond Anvil Cell, Rev. Sci. Instrum. 87, 076103 (2016).

37. Matsumoto, R. et al.Diamond anvil cells using boron-doped diamond electrodes covered with undoped diamond insulating layer, Appl. Phys. Express 11, 053101 (2018).

38. Matsumoto, R. et al.Pressure-Induced Superconductivity in Sulfur-Doped SnSe Single Crystal Using Boron-Doped Diamond Electrode-Prefabricated Diamond Anvil Cell, J. Phys. Soc. Jpn. 87, 124706 (2018).

39. Irifune, T. et al. Ultrahard polycrystalline diamond from graphite, Nature 421, 599 (2003).

40. Piermarini, G. J. et al. Calibration of the pressure dependence of the $R_{1}$ ruby fluorescence line to 195 kbar, Appl. Phys. 46, 2774 (1975).

41. Hammersley, A. P. et al. Two-dimensional detector software: From real detector to idealised image or two-theta scan, High Press. Res.14, 235 (1996).

42. Mao, H. K. \& Bell, P. M.High-pressure physics, The 1-megabar mark on the ruby $R 1$ static pressure scale, Science 191, 851 (1976).

43. Petricek, V., Dusek, M.\&Palatinus, L. Crystallographic Computing System JANA2006: General features, Z. Kristallogr. 229, 345 (2014).

44. Hämäläinen, K.et al. Elimination of the inner-shell lifetime broadening in x-ray-absorption spectroscopy, Phys. Rev. Lett. 67, 2850 (1991).

45. Hämäläinen, K. et al. Spin-dependent x-ray absorption of $\mathrm{MnO}$ and $\mathrm{MnF}_{2}$, Phys. Rev. B 46, 14274 (1992).

46. Yamaoka, H. Pressure dependence of the electronic structure of $4 f$ and $3 d$ electron systems studied by X-ray emission spectroscopy, High Press. Res. 36, 262 (2016).

\section{Figures}



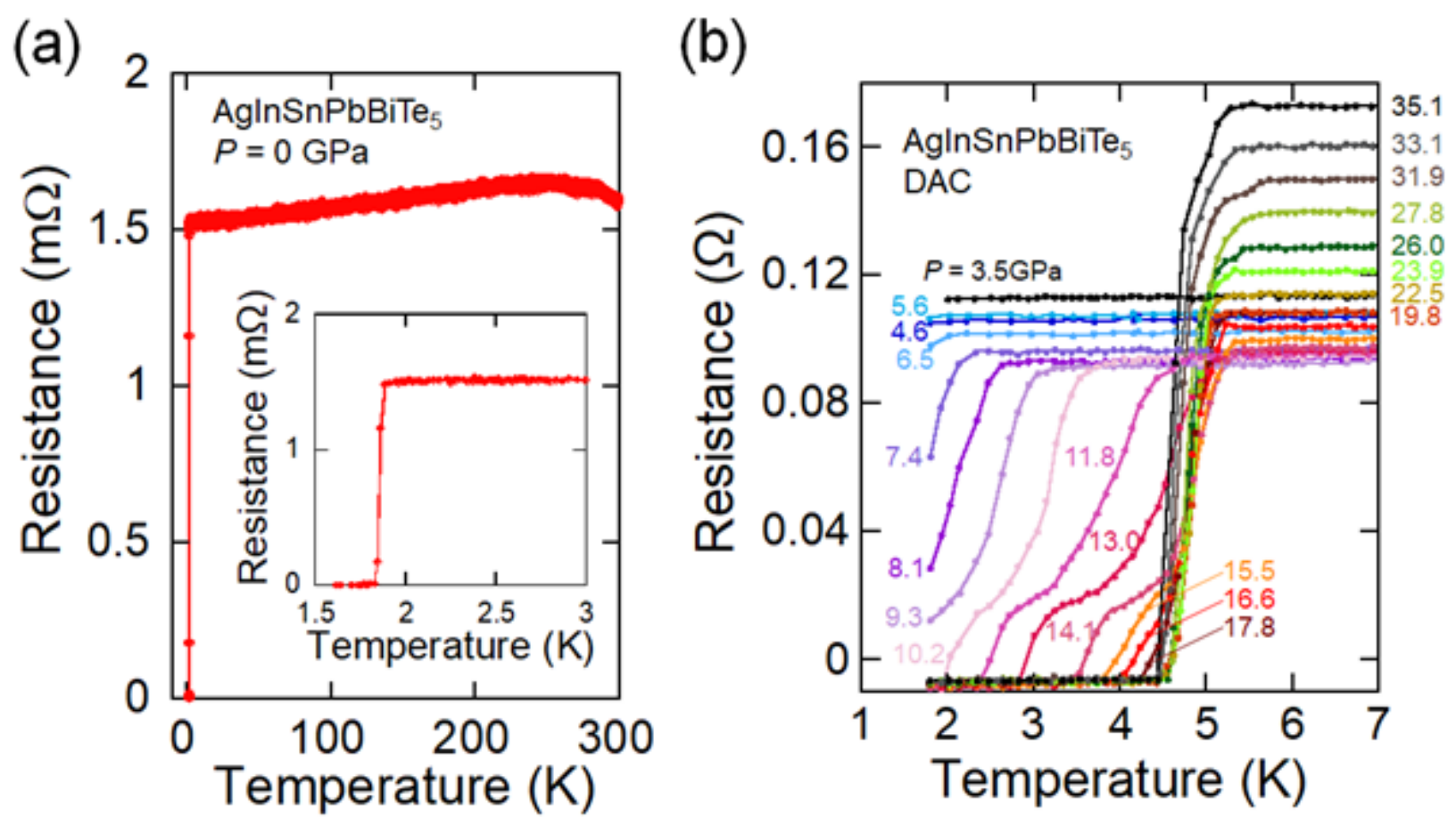

Figure 1

Superconducting transition in AgInSnPbBiTe $e_{5}$ under high pressure.a. Temperature dependence of electrical resistance of $\mathrm{AgInSnPbBiTe}_{5}$ measured using the conventional four-probe method.

b.Temperature dependences of electrical resistance of $\mathrm{AgInSnPbBiTe}_{5}$ measured using a diamond anvil cell (DAC). 
(a)

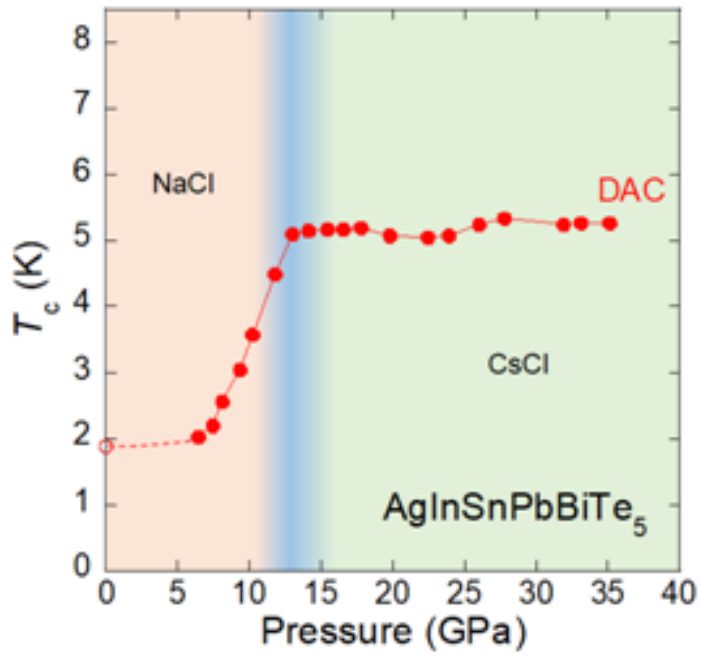

(b)

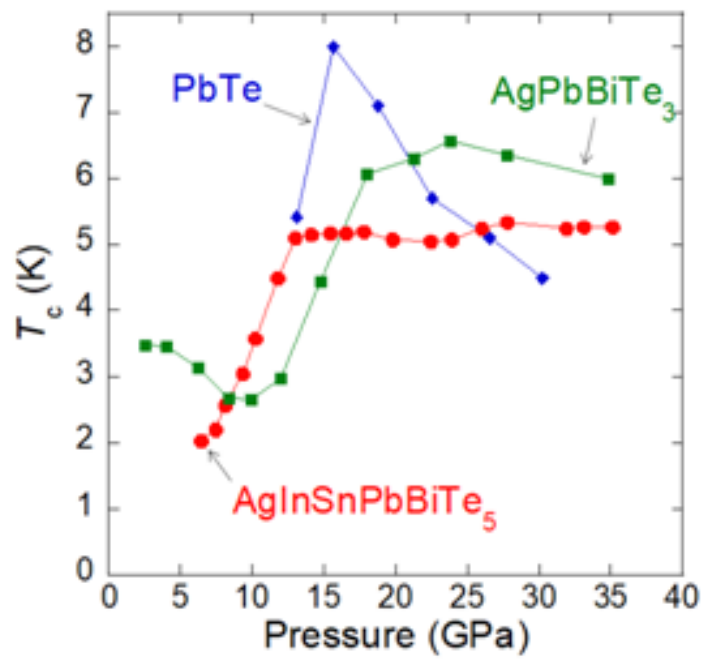

(c)

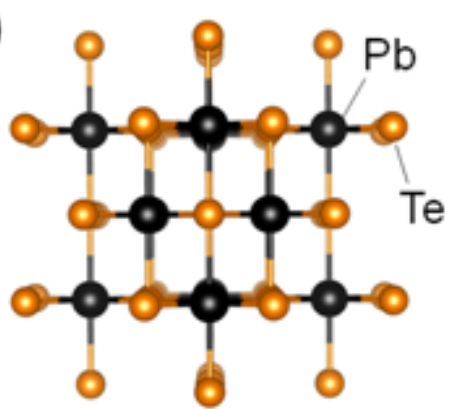

$\mathrm{PbTe}$

$\left(\Delta S_{\text {mix }}=0\right)$

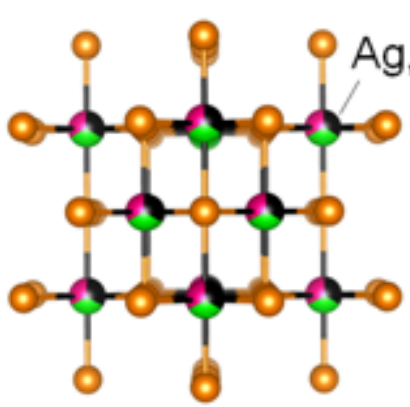

$\mathrm{AgPbBiTe}_{3}$

$\left(\Delta S_{\text {mix }}=1.1 R\right)$

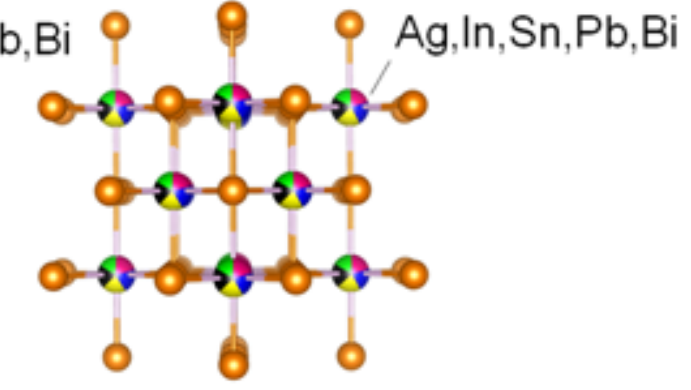

AgInSnPbBiTe ${ }_{5}$

$\left(\Delta S_{\text {mix }}=1.6 R\right)$

Figure 2

Pressure evolutions of $T_{\mathrm{c}}$ of metal tellurides with different configurational entropy of mixing. a. Pressure dependence of $T_{\mathrm{C}}$ of $\mathrm{AgInSnPbBiTe}$. Open and filled circles indicate the data taken without a pressure cell, and the data that was measured with DAC. The structural types are indicated according to structural analyses in Fig. 3d. b. Pressure dependences of $T_{\mathrm{c}}$ for $\mathrm{PbTe}, \mathrm{AgPbBiTe}_{3}$, and $\mathrm{AgInSnPbBiTe}{ }_{5}$.c. Schematic images of $\mathrm{NaCl}$-type crystal structure and configurational entropy of mixing ( $\left.\mathrm{D} S_{\text {mix }}\right)$ at the $M$ site of $\mathrm{PbTe}$, $\mathrm{AgPbBiTe}_{3}$, and AgInSnPbBiTe . $_{\text {. }}$

Figure 3

Pressure evolutions of crystal structure for metal tellurides with different configurational entropy of mixing.a-c. SXRD patterns for $\mathrm{PbTe}, \mathrm{AgPbBiTe}_{3}$, and $\mathrm{AgInSnPbBiTe}_{5}$. Note that the baseline height of the XRD pattern at each pressure scales to the pressure. $\mathbf{d}-\mathbf{f}$. Lattice volumes divided by $Z$ (chemical formula sum in a unit cell) for $\mathrm{PbTe}, \mathrm{AgPbBiTe}_{3}$, and $\mathrm{AgInSnPbBiTe}{ }_{5}$ are plotted as a function of pressure. In Fig. 
3d, the analysis results reported in Ref. 24 (Li et al.) are represented by orange lines. Structural types are shown in the figures.

(a)

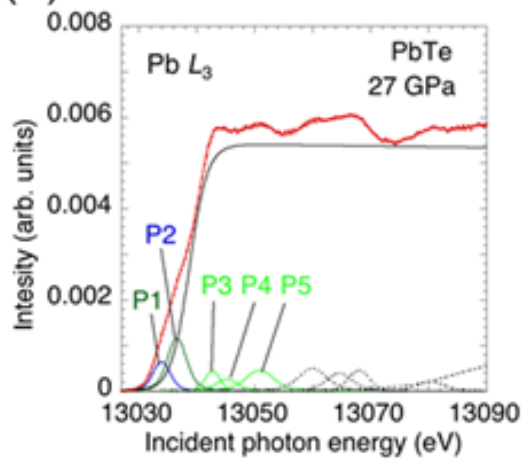

(d)

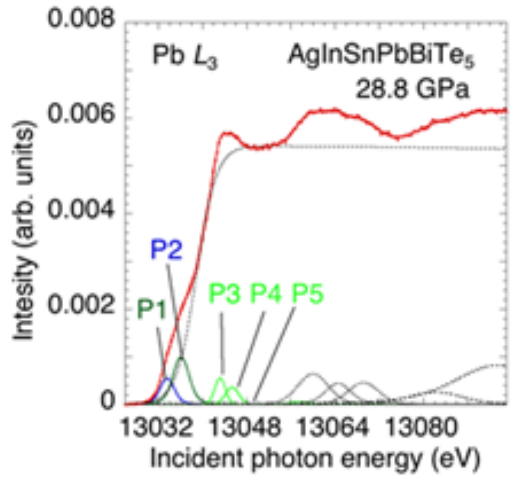

(b)

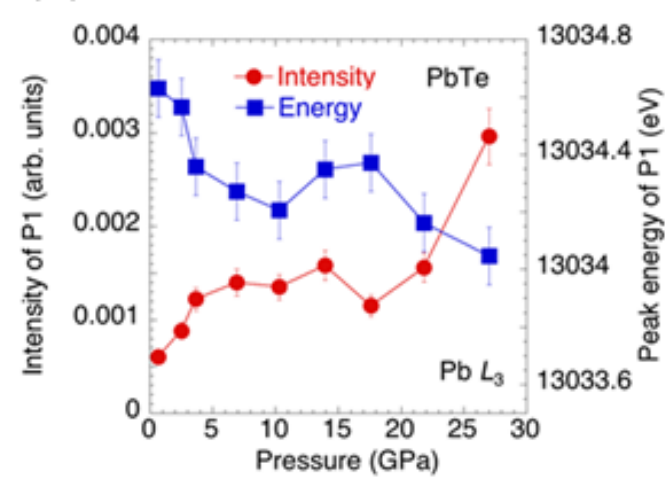

(e)

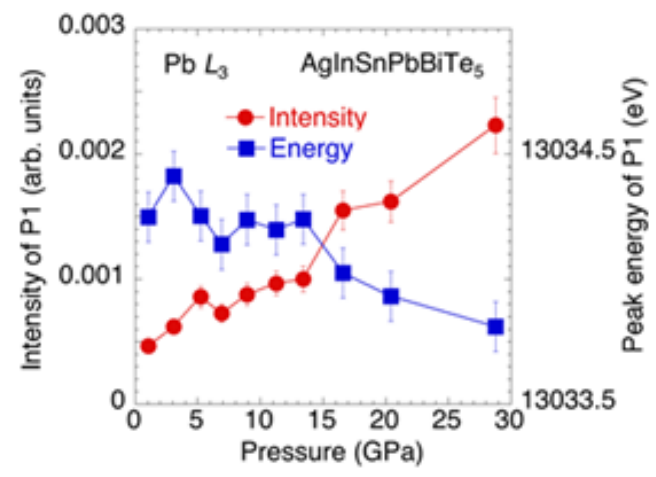

(c)

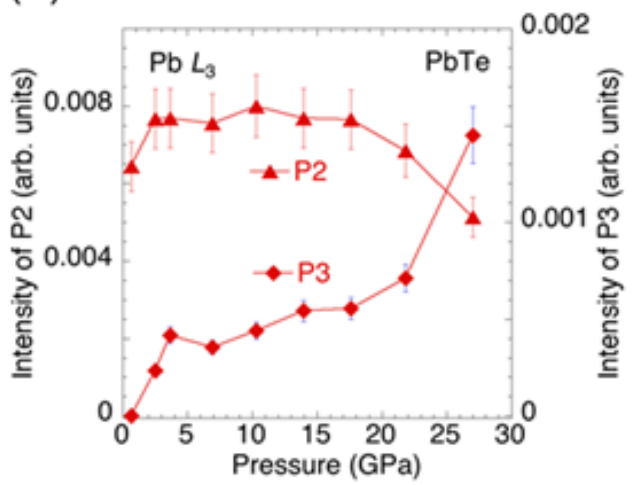

(f)

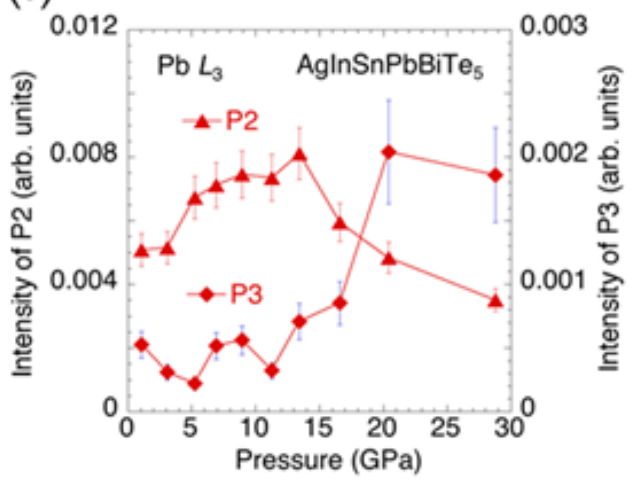

\section{Figure 4}

Electronic structure of $\mathrm{PbTe}$ and AgInSnPbBiTe ${ }_{5}$ under high pressure. a. Typical $\mathrm{Pb}-L_{3} \mathrm{PFY}-\mathrm{XAS}$ spectrum and an example of the fit to the spectrum for $\mathrm{PbTe}$ at $27 \mathrm{GPa}$. b. Pressure dependence of the intensity and the energy of the peak P1 for PbTe. c. Pressure dependence of the intensity of the peaks P2 and P3 for PbTe. d. Typical Pb- $L_{3}$ PFY-XAS spectrum and an example of the fit to the spectrum for $\mathrm{AgInSnPbBiTe}_{5}$ at $28.8 \mathrm{GPa}$. e. Pressure dependence of the intensity and the energy of the peak P1 for $\mathrm{AgInSnPbBiTe}_{5}$. f. Pressure dependence of the intensity of the peaks P2 and P3 for AglnSnPbBiTe ${ }_{5}$.

\section{Supplementary Files}

This is a list of supplementary files associated with this preprint. Click to download.

- SIKasemMTe20211209.pdf 